\title{
Association between Intercanine Width and Mandibular Dental Arch Forms
}

\author{
Saad Abdul Rehman, Sadia Rizwan, Syed Shah Faisal and Syed Sheeraz Hussain \\ Department of Orthodontics, Karachi Medical and Dental College, Karachi, Pakistan
}

\begin{abstract}
A descriptive cross-sectional study was conducted to investigate the association between intercanine width and the mandibular arch form in 109 dentate subjects, who visited Orthodontics Department from October 2018 to December 2019. Using Vernier caliper, the intercanine width was measured as the distance between the left and right canines on each cast with the arch form, also categorized as tapered, square or ovoid, using predefined criteria. Spearman's rank correlation demonstrated highly significant association between intercanine width and mandibular dental arch forms, with $\mathrm{p}$ value of 0.005 .
\end{abstract}

Key Words: Dentate subjects, Mandibular arch forms, Inter-canine width, Sexual dimorphism, Mandibular dental cast.

How to cite this article: Rehman SA, Rizwan S, Faisal SS, Hussain SS. Association between Intercanine Width and Mandibular Dental Arch Forms. J Coll Physicians Surg Pak 2021; 31(04):478-480.

The orthodontic treatment success depends on accurate diagnosis on orthodontic diagnostic casts. Most frequently considered measurements are: space analysis, anterior arch width, posterior arch width, intercanine distance, depth of curve of Spee, overbite, overjet, arch forms and arch width, especially intercanine width, remain few of the most important assessed parameters exerting a strong influence on the orthodontic treatment plan. There are multiple definitions of the inter-canine width reported by authors. ${ }^{1}$ The definition presented by Paulino et al. is "A linear distance between the cusps of contra-lateral canines or, in the case of presenting evidence of wear, the distance between the centres of the worn surfaces."

The dental arch form evaluation has become an important part of the orthodontic treatment planning. In fact, the study of dental arches is of great relevance to orthodontists; and the rightchoice of orthodontic wires may lead to a more stable dentition and decreasing the probability of dental relapse at the end of the treatment. A change in inter-canine width may represent a predictor of dental relapse. Therefore, it is fundamental to maintain the original arch dimension, choosing the right arch wire form during the treatment, and keeping the dentition in the soft tissue envelope. Various methods have been proposed, but two of them were used in most of the studies. One of the most common methods was used in this study, based on orthoform templates prepared by 3 M Unitek. ${ }^{3}$

Correspondence to: Dr. Saad Abdul Rehman, Department of Orthodontics, Karachi Medical and Dental College,

Karachi, Pakistan

E-mail: saadmemon_786@hotmail.com

Received: May 30, 2020; Revised: September 24, 2020;

Accepted: December 15, 2020

DOI: https://doi.org/10.29271/jcpsp.2021.04.478
It has been observed in a study by Nojima et al. that the Caucasian population has significantly lower intercanine width as compared to the Japanese population. There is a greater frequency of tapered and ovoid arch forms in Caucasian group, while Japanese group had more frequency of square arch forms. ${ }^{4}$ Maintenance of arch form and intercanine width is a vital step for long-term stability of the treatment. This study was conducted to gather information regarding intercanine width presented among orthodontic patients in local setup and its association with different mandibular arch forms.

This study was approved by the Ethical and Scientific Review Committee of Karachi Medical and Dental College (Ref No. 015/18). Non-probability consecutive method was used for sampling. By using WHO calculator on the basis of study of Mushtaq et al. ${ }^{5}$ taking statistics of mean intercanine width in mandible as $19.204 \pm 2.125$ in margin of error 0.4 at the confidence of interval 95\%, the calculated sample size was 109 . Mandibular dental casts of 109 patients, 33\% ( $\mathrm{N}=36)$ males with the mean age of $17.90 \pm 4.62$ years, and $67 \%(N=73)$ females with the mean age of $17.64 \pm 4.37$ years were obtained from the patients visited to the Department of Orthodontics. Inclusion criteria were patients of either gender seeking orthodontic treatment, must have permanent dentition in the arch and mandibular permanent canines erupted, aged 12 years or above. Exclusion criteria were individuals having missing or impacted permanent canines, missing teeth other than the third molars, any tooth anomaly of number, size, form and position as confirmed on clinical examination, having any known systemic disease, cleft lip and palate, and history of any facial trauma or surgical procedure of jaws and previous orthodontic treatment.

All the patients were examined by the researchers. Inter-canine width was measured from the cusp tips of left canine to the right 
canine cusp tip of mandibular dental cast with the help of digital Vernier caliper. The orthoform templates, prepared by $3 \mathrm{M}$ Unitek (Figure la), were overlaid on the mandibular casts taking care of the midline (Figure I-b). Arch forms were chosen according to the best fit method. To eliminate error of recording, the arch form of each case was judged two times by two examiners. All measurements were recorded in a pre-structured data collection proforma.

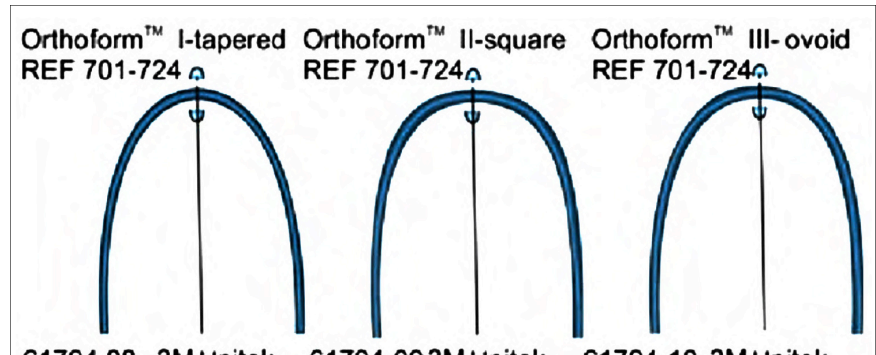

61794.08 3MUnitek 61794-093MUnitek 61794-10 3MUnitek

Figurela: Orthoform template by 3 MUnitek.

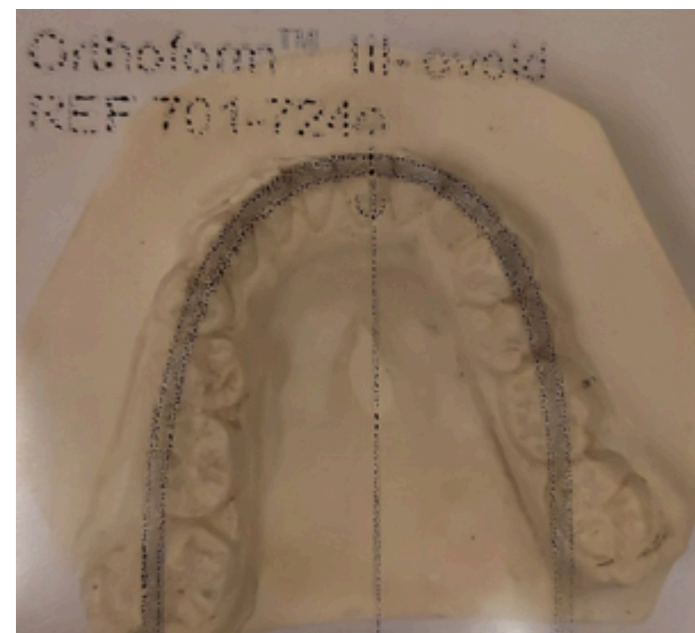

Figure lb: Arch form judgement Orthoform template by 3MUnitek.

The data were analysed statistically using software IBM SPSS Statistics version 20. Shapiro-Wilk's test was used to assess the normality of the data. The statistical analyses involved calculation of mean and standard deviation of age, median and interquartile range of inter-canine width of mandibular arches and frequencies along with percentages of qualitative variables like gender and groups of different arch forms. Mann-Whitney Utest has been used to find the difference in the value of intercanine width in genders. Kruskal-Wallis test was used to determine differences among arch forms along with post-hoc analysis for pairwise comparisons. Spearman's rank correlation test was used to determine the relationship between intercanine width and mandibular dental arch forms. A p-value of less than 0.05 was considered statistically significant.

The median value of mandibular intercanine width in males was 26.28 (24.70 - 29.88) mm; and in females, it was 26 (24.54 $27.67) \mathrm{mm}(p=0.030)$. Table I shows that out of 109 patients, $78 \%(\mathrm{~N}=85)$ had ovoid mandibular arch form, $11.9 \%(\mathrm{~N}=13)$ had square mandibular arch form, and $10.1 \%(\mathrm{~N}=11)$ had tapered mandibular arch form $(p=0.010)$. Pairwise analysis showed a significant difference between tapered and ovoid mandibular arch forms ( $p=0.009)$. Spearman's rank correlation demonstrated highly significant association between intercanine width and mandibular dental arch forms with the correlation value of $r=0.268$ and $p=0.005$.

Table I: Median values of intercanine width in the various arch forms.

\begin{tabular}{|l|l|l|l|}
\hline Arch forms & $\mathbf{N}(\%)$ & $\begin{array}{l}\text { Median values }(\mathbf{m m}) \text { of } \\
\text { intercanine width }\end{array}$ & p-value \\
\hline Tapered & $11(10.1)$ & $24(22.65-25)$ & \multirow{2}{*}{0.010} \\
\hline Square & $13(11.9)$ & $26(23-28.5)$ & \\
\hline Ovoid & $85(78)$ & $26.4(25-28.65)$ & \\
\hline
\end{tabular}

According to multiple studies done by Ferro et al. ${ }^{6}$ and others, in regard of the mandibular dental arch, males had a higher intercanine width in comparison to the females; but the intercanine width was similar in the three arch shapes and no significant difference was detected unlike this study, which showed significant difference in intercanine width among three different dental arch forms. In literature, it has been observed that inter-canine width increased as the mandibular arch form changed from square to ovoid to tapered. This finding is in contradiction to the findings of the present study. Out of three arch forms, ovoid arch form showed significant differences in intercanine width. The most common type of mandibular arch was ovoid, followed by square and tapered arch forms. Significant differences found in the values of intercanine width among three groups of mandibular arch forms.

Larger sample size and mathematical ways to define the arch form can be used to enhance the outcome of the research. From this study, intercanine width was concluded to be the key factor in determining the arch form.

\section{ETHICALAPPROVAL:}

Ethical and Scientific Review Committee of Karachi Medical and Dental College (Reference No.015/18).

\section{PATIENTS' CONSENT:}

Written informed consents were obtained from patients who participated in thisstudy.

\section{CONFLICT OF INTEREST:}

All authors declared no conflict of interest.

\section{AUTHORS' CONTRIBUTION:}

SAR: Conception and design of the study, the acquisition of data, or the analysis and interpretation.

$\mathrm{SR}$ : Critical revision of the article.

SSF: Helped and supervised the project and also provided critical revision of the article.

SSH: Helped in the conception and design of the study, and gave final approval of the version to publish.

All authors discussed the results and contributed to the final manuscript.

\section{REFERENCES}

1. Azeem M, Haq A, Qadir S. Maxillary intercanine widths. Prof Med J 2018; 25(02):246-51.

2. Paulino V, Paredes V, Gandia JL, Cibrian R. Prediction of 
arch length based on intercanine width. Eur J Orthod 2008; 30(3):295-8. doi: 10.1093/ejo/cjm115.

3. Mushtaq N, Tajik I, Baseer S, Shakeel S. Intercanine and intermolar widths in angle class i ii and iii malocclusions. Pak Oral Dental J 2014; 34(1).

4. Nojima K, McLaughlin RP, Isshiki Y, Sinclair PM. A comparative study of caucasian and japanese mandibular clinical arch forms. Angle Orthod 2001; 71(3):195-200. doi:
10.1043/0003-3219(2001)071<0195:ACSOCA>2.0.CO;2.

5. Saleem T, Shafique A. Mandibular arch form analyses: A comparison between results of two different methods. Pak Oral Dent J 2012; 32(3): 556-60.

6. Ferro R, Pasini M, Fortini A, Arrighi A, Carli E, Giuca MR. Evaluation of maxillary and mandibular arch forms in an Italian adolescents sample with normocclusion. Euro J Paediatr Dent 2017; 18(3):193-8. doi: 10.23804/ejpd. 2017.18.03.05. 